\title{
SEGMENTATION AND RECOGNITION OF HaNdWritTEn Digit Numeral STRING Using a Multi Layer Perceptron Neural Networks
}

\author{
N. Venkateswara Rao ${ }^{1}$ and Dr. B. Raveendra Babu ${ }^{2}$ \\ ${ }^{1}$ Dept. of Computer Science \& Engineering, R.V.R. \& J.C. College of Engineering, \\ Guntur, INDIA \\ ${ }^{2}$ Professor, Dept. of Computer Science \& Engineering VNR Vignana Jyothi Institute of \\ Engineering and Technology, Hyderabad, INDIA
}

\begin{abstract}
In this paper, the use of Multi-Layer Perceptron (MLP) Neural Network model is proposed for recognizing unconstrained offline handwritten Numeral strings. The Numeral strings are segmented and isolated numerals are obtained using a connected component labeling $(C C L)$ algorithm approach. The structural part of the models has been modeled using a Multilayer Perceptron Neural Network. This paper also presents a new technique to remove slope and slant from handwritten numeral string and to normalize the size of text images and classify with supervised learning methods. Experimental results on a database of 102 numeral string patterns written by 3 different people show that a recognition rate of $99.7 \%$ is obtained on independent digits contained in the numeral string of digits includes both the skewed and slant data.
\end{abstract}

\section{KEYWORDS}

Connected Components Labeling, Multi-Layer Perceptron Neural Networks, Segmentation, Feature Extraction, Handwritten recognition.

\section{INTRODUCTION}

Recognizing writer-independent handwritten numeral string of digits is still a difficult problem for a computer although it has been a research topic for over so many decades. The key problem is due to an unlimited number of styles, sizes and variations of digit patterns used by different people. As a critical preprocessing stage in handwritten numerical string recognition, the numerical string digits segmentation exports results which will affect the performance of the overall recognition system. There still exist several challenges in the numerical string segmentation for hand written document. Unlike machine printed document, the free-style handwritten numerical string of digits is often curved, have various skew angles, no uniform direction, connect or overlap with each other. A study of the present literature exposes that an excessive amount of research work has been made to resolve the problem. Different sets of features in topological and spatial domain, as well as in frequency domain, have been proposed for the recognition of handwritten characters [1]. Moreover, a variety of classification methods, such as template matching, structural, syntactic and neural network approaches are adopted for 
classification and/or recognition [2, 11]. However, most of these algorithms could not yield satisfactory recognition performance in the cases of rotated and noisy patterns. Multi-module (multi-expert) neural networks have been studied for the last few years in order to reduce the learning complexity and to improve the system performance [3]. The neural network classifiers are generally classified into two categories. In one category, each module of the neural network is used to look after a sub-region of the feature space or a subgroup of classes in the hope that each of these local expert can take better care of its own domain so that the overall performance can be improved $[4,5]$. In the other category, each module of the neural network is used to deal with the input from one of the multiple sensors that are used to collect all possible data. Handwritten character recognition task is very complex. Normally recognition of isolated characters is a simple task than a string of characters [6-10].These systems are used in many areas like automatic processing of bank checks, tax forms, postal identification numbers, optical response sheets etc.,. In this work, a connected component labeling algorithm [12] is used to segment the individual numerals of the image. For any character recognition problems to segment the characters, it is required to do some preprocessing methods for getting better features.

\section{SEgMENTATION AND RECOGNITION OF HAND WRITTEN NUMERAL STRING}

The procedure done before processing by correcting images from different errors is called preprocessing. The preprocessing is to be done before image enhancement. It includes conversion to a binary image, applying Median filtering to remove noise, and Thinning etc.

\subsection{Binarization:}

Scanned input image is given as input. It is checked whether the image is color or grayscale or binary. If the image is not binary image it is converted to binary image.

1. Calculating the size of the image.

2. Finding the sum of the pixels.

3. Calculating the average threshold value using the sum of the pixels and the size of the image.

4. Comparing the generated threshold value with the pixel value of the image. If the pixel is value is greater than the threshold value, then assign the pixel a value of ' 1 ' otherwise ' 0 '.

\subsection{Noise Removal:}

Median filters are commonly used methods to remove the noise. Median filter is one of the most popular non-linear filters to remove the salt \& pepper noise. The noise is removed by substituting the mask center value by the median value of midpoint neighborhood.

\subsection{Skew Correction}

In mathematical terms skew means, lines that are neither parallel nor intersecting. The skew correction is performed on such lines. The image is rotated with an angle to remove the skew. Skew correction is used to align the image base line with the x-axis.In this system, a lower baseline is drawn where the maximum pixels are located. An example is shown in Figure 1. 
1) The lowest black pixel is determined in every column of the image to populate the set $\mathrm{S}$.

$$
S=\{s i=(x i ; y i) / \text { lowest black pixel in column } x i\}
$$

2) To fit as the baseline of the current line, the data set $S$ is used to calculate a least-squares linear regression to find a base line of the form $y=m x+c$

3) Computing the arctangent of the line slope that produces the rotation angle, $\operatorname{theta}=\arctan (\mathrm{m})$

4) Rotate the image by negative theta value computed in step 3 to remove the skew.
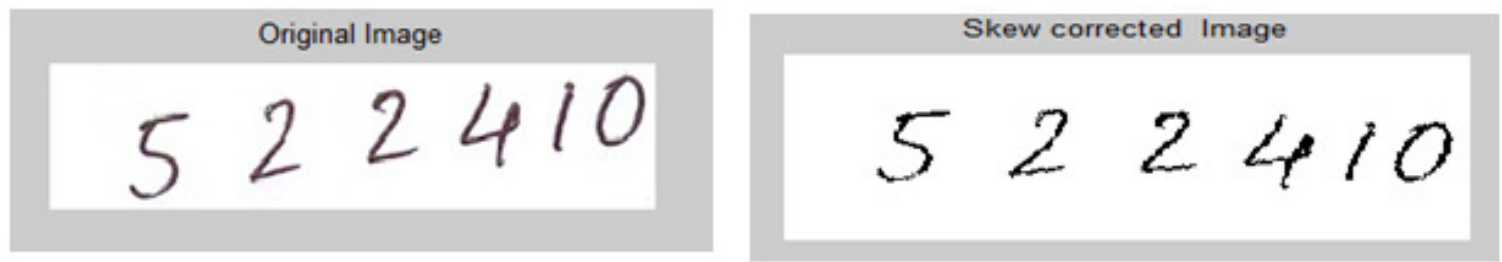

Figure 1 Sample Skew Correction Image

\subsection{Slant Removal}

It is used to normalize writing where the text is at an angle with the goal of making the text upright. Here based on a line, image is rotated with the angle so that the slant present in the image is removed.

The slant correction algorithm is as follows:

1) Calculate the new transformations from -45 degrees to 45 degrees angle to shear and transform the line

2) Generate a vertical projection histogram for each shear angle calculated in the above step.

3) Calculate Time Frequency distribution of each vertical histogram computed in the above step.

4) Remove the slant of the image by choosing angle representing the largest distribution intensity from the histogram computed in the above step.
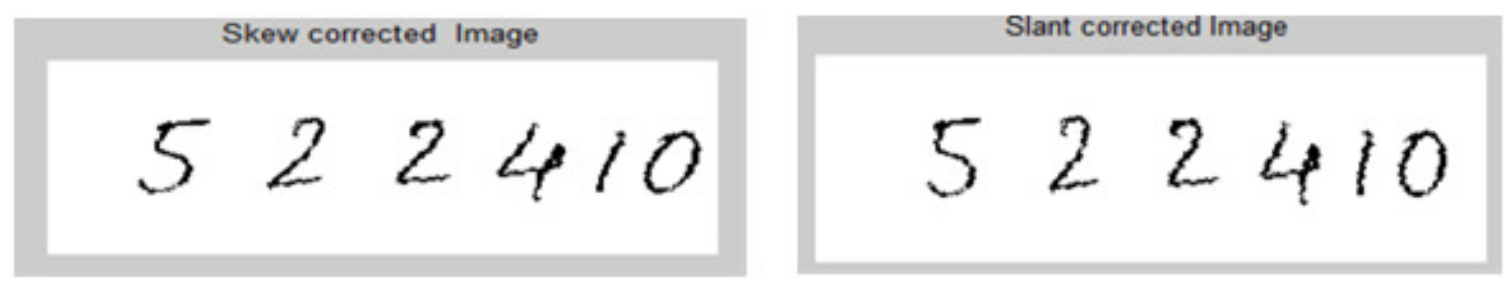

\subsection{Segmentation}

Figure 2 Sample Slant Correction Image

Segmentation is the process of dividing the string of numeral digits into individual digit images. Normally there are three approaches for dividing the string of numerals into individual digits. They are 1) External segmentation, where digit boundaries are found prior to segmentation, 2) Internal segmentation, in which letter boundaries are determined as part of recognition, and 3) No segmentation, where recognition occurs at string level. In this paper an internal segmentation is done by using a connected components labeling (CCL) approach method. 
The algorithm for finding the connected components of an image is

1) Scan the image by moving along a row until it comes to a point $p$ (where $p$ denotes the pixel to be labeled at any stage in the scanning process) for which $V=\{1\}$.

2) When this is true, examine the four neighbors of $p$ which have already been encountered in the scan
a. To the left of $p$.
b. Above it.
c. The two upper diagonal terms.

3) The labeling of $p$ occurs as follows:

a. If all four neighbors are 0 , assign a new label to $p$, else

b. if only one neighbor has $V=\{1\}$, assign its label to $p$, else

c. If more than one of the neighbors have $V=\{1\}$, assign one of the labels to $p$ and make a note of the equivalences.

\subsection{Feature Extraction}

For achieving high recognition rate, the selection of appropriate feature extraction method is very important. After pre-processing i.e., skew correction, slant correction, and segmentation is done the image is normalized to a $15 \times 15$ without changing the aspect ratio. A total of 225 features are taken from the image to classify the image. The features of the characters that are important for classifying them at recognition stage are extracted. This is an important stage as its effective functioning increases the recognition rate and decreases the misclassification. The features are extracted for all images after segmenting the string of numerals into individual digits where one sample of the image is shown in Figure 1 which is a skew corrected image and Figure 2 is after slant correction.

\subsection{Classification}

As the features are extracted, an appropriate classifier must be selected. A number of classifiers are used and each classifier suitable to classify a specific kind of feature vector depending upon its characteristics is found. The Neural Network classifier is used commonly for classification. A multi-layer perceptron neural network classifier is used here for recognition. The most common classifier model is multi-layer perceptron neural networks. In order to learn this neural network, it requires a desired output because it is a supervised network. The goal of this network is to create a model which correctly maps from input to the output with historical data. A sample representation of a multi-layer perceptron is shown in Figure 3. 


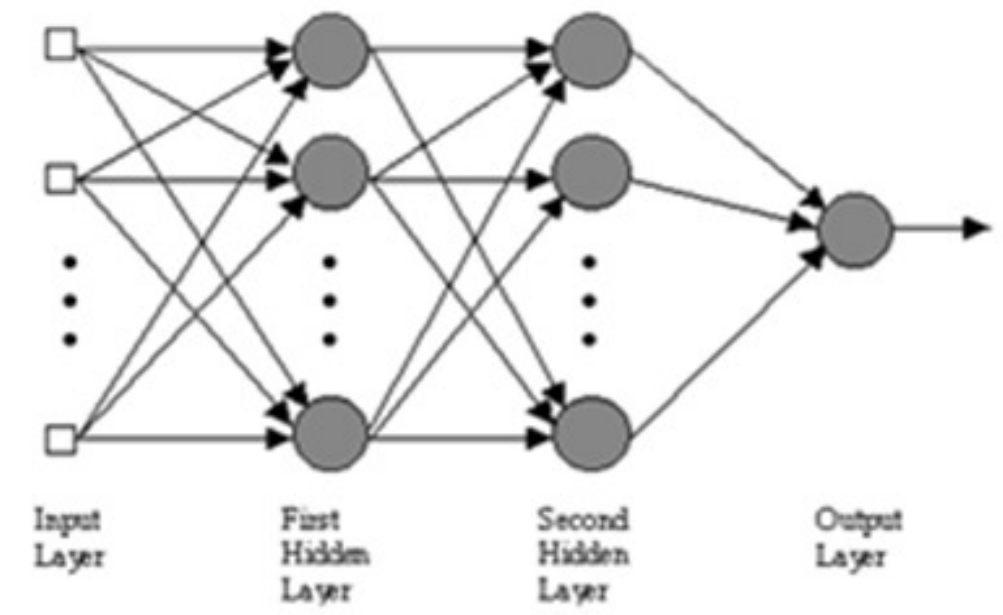

Figure 3 Architecture of Multi Layer Perceptron (MLP) Neural Network

\section{RESUltS AND DiscuSSIONS}

Experiments are conducted on different numerical string of characters written by three different writers. In this work, 40 samples from writer 1 and 38 samples from writer2 and 24 samples from writer3 are collected. A total of 102 numerical strings of characters from these three different writers are collected. These samples contain a total number of 617 individual digits covering all the digits approximately equal which is shown in table 1. Each sample of numerical string is segmented into individual digits. Each digit is normalized to a size of $15 \mathrm{X} 15$ pixels. From the normalized image the features are extracted and training is performed with the multi layer perceptron back propagation neural network. From the confusion matrix, shown in Figure 4, it is observed that a recognition rate of $99.7 \%$ and an error rate of $0.3 \%$ is obtained. Some of the sample images written by different writers are shown in Figure 5.

Table 1 Sample Images database

\begin{tabular}{ccccc}
\hline & Writer1 & Writer2 & Writer3 & Total \\
\hline No: of samples & 40 & 38 & 24 & 102 \\
Number of 0's & 22 & 21 & 14 & 57 \\
Number of 1's & 29 & 22 & 15 & 66 \\
Number of 2's & 28 & 22 & 15 & 65 \\
Number of 3's & 19 & 23 & 17 & 57 \\
Number of 4's & 25 & 22 & 15 & 62 \\
Number of 5's & 25 & 24 & 12 & 63 \\
Number of 6's & 27 & 24 & 14 & 65 \\
Number of 7's & 26 & 22 & 14 & 62 \\
Number of 8's & 27 & 22 & 15 & 63 \\
Number of 9's & 23 & 21 & 13 & 59 \\
\hline Total Digits & 251 & 223 & 144 & 617 \\
\hline
\end{tabular}




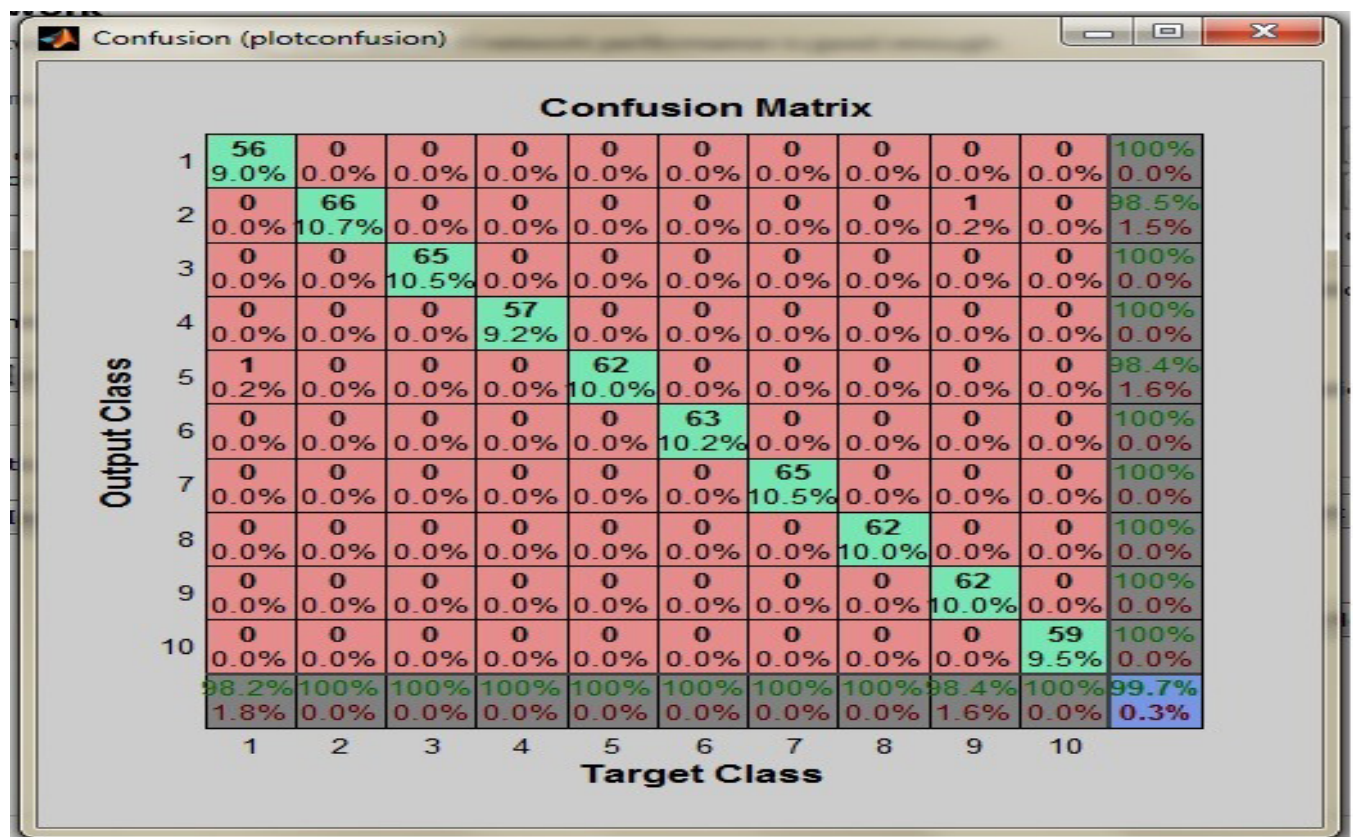

Figure 4 Confusion Matrix
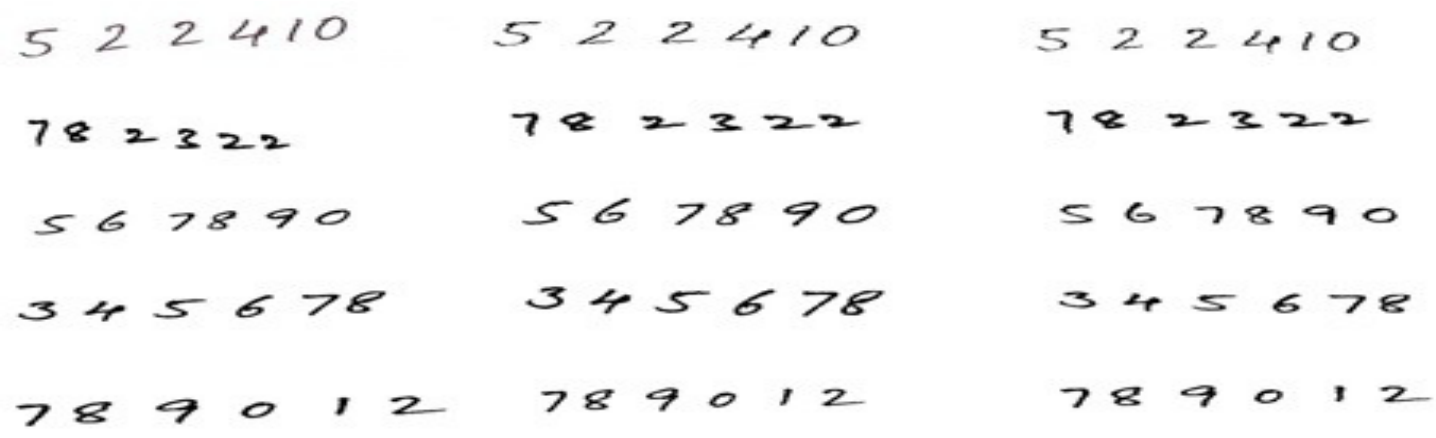

Figure 5 Sample Images

\section{Conclusion}

Previous work of the authors of this paper is limited to an isolated digit character. In this paper, a new method for handwritten digits recognition from a string of numerals using a multi-layer perceptron neural network is presented. The effectiveness of this proposed method was evaluated by computing the recognition rate and error rate. It is observed that a recognition rate of $99.7 \%$ and an error rate of $0.3 \%$ on handwritten string of numerals is obtained.

\section{REFERENCES}

[1] C. Y. Suen. Distinctive features in automatic recognition of hand printed characters. Signal Processing, 4(2 \& 3):193 - 207, 1982.

[2] S. Mori, C. Y. Suen, and K. Yamamoto. Historical review of OCR research and development. Proceedings of the IEEE, 80(7):1029 - 1058, 1992. 
[3] Michael I. Jordan and Robert A. Jacobs. Hierarchies of adaptive experts. In Advance in Neural Information Processing Systems 4,

[4] Tatsuo Matsuoka, Hiroshi Hamada, and Ryohei Nakatsu. Syllable recognition using integrated neural networks. In IEEE INNS International Joint Conference on Neural Networks, volume 1, 1989.

[5] Z. Chi and M. Jabri. A Multi-Module Neural Network Approach for ICEG Classification. In Proceedings of the Third Australian Conference on Neural Networks, Canberra, Australia, 1992.

[6] Thien M. Ha, Matthias Zimmermann, Horst Bunke, Off-line handwritten numeral string recognition by combining segmentation-based and segmentation-free methods, Journal of Pattern Recognition, Volume 31, Issue 3, Pages 257-272, March 1998.

[7] Zhixin Shi, Venu Govindaraju, Segmentation and recognition of connected handwritten numeral strings, Journal of Pattern Recognition, Volume 30, Issue 9, Pages 1501-1504, 1997.

[8] Ashraf Elnagara, Reda Alhajj, Segmentation of connected handwritten numeral strings, Journal of Pattern Recognition, Volume 36, Issue 3, Pages 625-634, March 2003.

[9] Yi-Kai Chen, Jhing-Fa Wang, Segmentation of single- or multiple-touching handwritten numeral string using background and foreground analysis, IEEE Transactions on Pattern Analysis and Machine Intelligence, Volume 22, Issue 11, Pages 1304 - 1317, Nov 2000.

[10] Javad Sadria, Ching Y. Suena, Tien D. Bui, A genetic framework using contextual knowledge for segmentation and recognition of handwritten numeral strings, Journal of Pattern Recognition, Volume 40, Issue 3, Pages 898-919, March 2007.

[11] N. Venkateswara Rao, Dr. B. Raveendra Babu, G. Rama Mohan Babu, A Radial Basis Function Neural Network to Recognize Handwritten Numerals with normalized moment features from skeletons, IEEE International Conference on Recent Trends in Information Technology (ICRTIT), Pages 68 - 72, 2013.

[12] Di Stefano, L.Bulgarelli, Andrea , A simple and efficient connected components labeling algorithm, International Conference on Image Analysis and Processing, Pages 322 - 327, 1999.

\section{Authors}

N. VenkateswaraRao, received his M.Sc degree in Computer Science Department from Acharya Nagarjuna University, India. He did his M.Tech in Computer Science \& Technology from Andhra University, India. He is currently working as Associate Professor, in the Department of Computer Science \& Engineering at RVR \& JC College of Engineering, Guntur, India. He has 15 years of teaching experience. His research areas of interest include Artificial Neural Networks, Image Processing, and Pattern Recognition. He is life member of ISTE.

Dr. B. Raveendra Babu, obtained his Masters in Computer Science and Engineering from Anna University, Chennai. He received his Ph.D. in Applied Mathematics at S. V. University, Tirupati. He is currently working as professor and HOD in department of Computer Science \& Engineering at VNR Vignana Jyothi Institute of Engineering and Technology, Hyderabad. He has 30 years of teaching experience. He has more than 40 international \& national publications to his credit. His research areas of interest include VLDB, Image Processing, Pattern analysis and Wavelets. He is life member in professional bodies like ACM, ISTE and CSI.
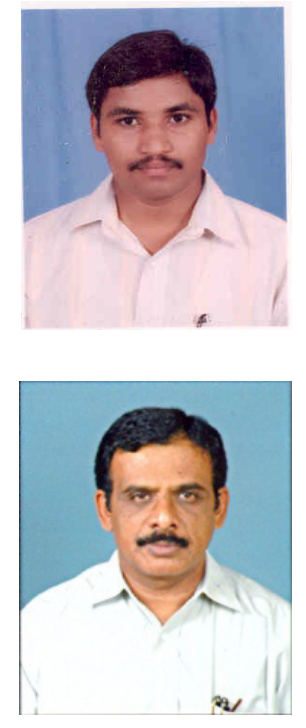